\title{
Heat and Mass Transfer Analysis in Chemically Reacting Flow of Non-Newtonian Liquid with Local Thermal Non-Equilibrium Conditions: A Comparative Study
}

\author{
A. Alhadhrami ${ }^{1}\left(\mathbb{D}\right.$, B. M. Prasanna ${ }^{2, *(D)}$, Rajendra Prasad K. C. ${ }^{3} \mathbb{D}$, K. Sarada ${ }^{4}$ and Hassan A. H. Alzahrani ${ }^{5}$ \\ 1 Department of Chemistry, College of Science, Taif University, P.O. Box 11099, Taif 21944, Saudi Arabia; \\ a.babatin@tu.edu.sa \\ 2 Department of Chemistry, Jain Institute of Technology, Davanagere 577003, India \\ 3 Department of Mathematics, Jain Institute of Technology, Davanagere 577003, India; \\ rajendraprasadkp@gmail.com \\ 4 Department of Mathematics, Government City College, Hyderabad 500002, India; siddisarada@gmail.com \\ 5 Department of Chemistry, College of Science and Arts at Khulais, University of Jeddah, P.O. Box 355, \\ Jeddah 21921, Saudi Arabia; hahalzahrani@uj.edu.sa \\ * Correspondence: drbmprasanna@jitd.in
}

\section{check for} updates

Citation: Alhadhrami, A.; Prasanna, B.M.; K. C., R.P.; Sarada, K.; Alzahrani, H.A.H. Heat and Mass Transfer Analysis in Chemically Reacting Flow of Non-Newtonian Liquid with Local Thermal Non-Equilibrium Conditions: A Comparative Study. Energies 2021, 14, 5019. https://doi.org/10.3390/ en14165019

Academic Editor: Victor Becerra

Received: 8 July 2021

Accepted: 8 August 2021

Published: 16 August 2021

Publisher's Note: MDPI stays neutral with regard to jurisdictional claims in published maps and institutional affiliations.

Copyright: (c) 2021 by the authors. Licensee MDPI, Basel, Switzerland. This article is an open access article distributed under the terms and conditions of the Creative Commons Attribution (CC BY) license (https:/ / creativecommons.org/licenses/by/ $4.0 /)$.

\begin{abstract}
In the current paper, we endeavour to execute a numerical analysis in connection with the boundary layer flow induced in a non-Newtonian liquid by a stretching sheet with heat and mass transfer. The effects of chemical reactions and local thermal non-equilibrium (LTNE) conditions are considered in the modelling. The LTNE model is based on energy equations, and provides unique heat transfer for both liquid phases. As a result, different temperature profiles for both the fluid and solid phases are used in this work. The model equation system is reduced by means of appropriate similarity transformations, which are then numerically solved by employing the classical Runge-Kutta (RK) scheme along with the shooting method. The resultant findings are graphed to show the effects of various physical factors on the involved distributions. Outcomes reveal that Jeffrey fluid shows improved velocity for lower values of porosity when compared to Oldroyd-B fluid. However, for higher values of porosity, the velocity of the Jeffery fluid declines faster than that of the Oldroyd-B fluid. Jeffery liquid shows improved fluid phase mass transfer, and decays more slowly than Oldroyd-B liquid for higher values of chemical reaction rate parameter.
\end{abstract}

Keywords: local thermal non-equilibrium; porous medium; chemical reaction; Oldroyd-B and Jeffrey fluids

\section{Introduction}

To characterize heat transfer in porous media, two fundamental models may be used: the LTNE model (two-equation model), and the local thermal equilibrium (LTE) model (one-equation model). The thermal variance between the liquid and solid phases is accounted for in the LTNE model. In certain cases, the assumption of LTE is not valid if the temperature difference between the two phases is significant. At this point, the LTNE model must be employed, and an additional thermal boundary condition at the interface must be given. When the heat flux is large and the flow is fast, the effects of LTNE become considerable. In recent years, applications of nanofluids and porous media theories have made LTNE model research increasingly significant. Nield and Bejan [1] developed the first two-field model for the energy equation. To characterize the typical thermal behaviour of saturated media, they employed dual independent equations for the solid and fluid phases, rather than a single energy equation. Kuznetsov and Nield [2] used the Brinkman model to explore the LTNE effect on the onset of convection in nanoliquids. Sheremet et al. [3] coupled the LTNE with a Tiwari-Das nanoliquid model to replicate natural convection in a square cavity filled with a nanoliquid. Prakash et al. [4] inspected the non-Darcian 
flow of liquid on an upright stretched plate encased in a porous medium using the LTNE model. Mahajan and Sharma [5] demonstrated the consequences of LTNE on convection in magneto-nanoliquids.

The study of heat transfer is one of the most significant and commonly utilized engineering disciplines, and its relevance is heightened by the need for energy management and energy savings. Finding efficient ways to minimize energy usage and boost productivity is critical on this route. Optimizing heat transfer equipment to achieve improved energy efficiency necessitates a focus on equipment downsizing on the one hand, and increasing heat transfer per unit of area on the other. Because of its structure, a porous medium offers a significant heat transfer area in a given volume. As a result, research into heat transport in porous media has gained prominence in recent years. A porous medium is a solid substance with pores within it, and fluid flows through these continuous pores. Numerous studies have been conducted on heat transference in porous media, which is employed in many applications, such as cooling electronic components, combustion chambers, compact heat exchangers, researching granular bed reactors, and storing nuclear and radioactive waste. Muthtamilselvan et al. [6] explored the LTNE conditions on a laminar flow of nanofluid passing through an upright plate with a porous medium. Kumar et al. [7] demonstrated the Darcy flow of nanoliquid on a cylinder with radiative heat flux. Xiong et al. [8] elucidated the cross-nanofluid stream on a needle with a porous medium. Sharma and Mahajan [9] explored the influence of LTNE on the magnetic nanofluid layer in a permeable medium with magnetic field impact. Gowda et al. [10] reported dusty hybrid nanoliquid flow through a stretchy surface with a permeable medium.

The relationships of mass transfer and chemical reaction are extremely tough to study, and may be examined in the usage of reactant species and production at various rates within the mass transfer of fluid. Chemical reaction in a flow initiated by a stretched sheet is important in biochemical engineering, plastic extrusion, and metallurgy. A few instances include moisture and temperature supplies across an agricultural plot, or energy transmission in a rainy cooling tower. Many researchers' work has been examined in connection to these applications. Song et al. [11] elaborated the chemically reactive stream of a hybrid nanofluid with radiation in the presence of double diffusion. Khan et al. [12] elucidated the entropy analysis in the chemically reactive flow of a nanofluid with Marangoni convection and activation energy. Gowda et al. [13] modelled and discussed a chemically reactive flow of second-grade nanoliquid using Marangoni convection. Kumar et al. [14] exemplified the chemically reactive flow of Casson nanoliquid. Gowda et al. [15] elucidated the magnetic dipole impact on nanofluid flow over a stretching surface with chemical reaction.

Because of their importance in engineering and industry, it is critical to explore the flow behaviour of non-Newtonian liquids. This interest has grown as a result of their applications in biorheology, geophysics, petroleum and chemical industries. Some mathematical concerns concerning the boundary and beginning conditions remain unresolved; this is because the model equations for non-Newtonian liquids have a higher order than the Navier-Stokes equations. As a result, any study involving the closed-form solutions of nonNewtonian liquids constantly draws the attention of researchers. In non-Newtonian liquid models, Jeffrey and Oldroyd-B liquids have considerable applications in fluid mechanics due to their relaxing properties; hence, various researchers have performed comparative investigations on these fluid flow models past various surfaces. Sandeep et al. [16] used the Buongiorno model to compare Maxwell, Oldroyd-B, and Jeffrey fluid flows on a porous, stretchable sheet. Sandeep and Sulochana [17] proposed a unique model for examining the heat transfer in Oldroyd-B, Maxwell, and Jeffrey nanofluids on a stretchable sheet. Recently, Mabood et al. [18] explained the impact of heat generation on the radiative flow of Oldroyd-B liquid past a sheet, and discussed the flow and heat transport behaviour. Ibrahim and Gadisa [19] used the Galerkin finite-element method to discuss the convective heat transference in the flow of Oldroyd-B liquid on a sheet. Khan et al. [20] explained the bioconvective slip flow of Oldroyd-B liquid on a sheet with suspended nanoparticles and gyrotactic microbes on a sheet. Ali et al. [21] used the finite-element technique to discuss 
the impact of double diffusion on Oldroyd-B liquid flow with suspended nanoparticles on a sheet, and explained the convective heat and mass transport. Pandikunta et al. [22] deliberated the radiative dissipating flow of Jeffrey nanoliquid on a sheet, and discussed the convective heat transport. Shahzad et al. [23] explored the dissipative magnetized flow of Jeffrey nanomaterial liquid on a surface. Rasool et al. [24] explained the magnetized flow of Jeffrey liquid with a nanoparticle suspension on a sheet. Rasheed et al. [25] explicated the convective and radiative streams of Jeffrey liquid with magnetic effect past a sheet.

The analysis of boundary layer flow problems involving real fluids past stretching sheets/surfaces received considerable attention in various fields of science and engineering, including chemical engineering, plastic drawing, and polymer sheet extrusion. In connection to these, numerous researchers explored the flow of different liquids past a stretching sheet. Ishak et al. [26] numerically analysed the heat transfer in the flow of a liquid past a stretchable sheet using a finite-difference scheme. Vajravelu et al. [27] used the Keller box method to discuss the heat transport analysis of a viscous liquid past a stretchable sheet. Recently, Kumar et al. [28] explored the magnetic dipole and thermophoretic effects on Maxwell nanoliquid flow on a stretchable sheet. Gowda et al. [29] deliberated the heat and mass transport analysis of nanoliquid flow past a stretchable sheet. Mallikarjuna et al. [30] elaborated the dissipative flow of a dusty hybrid nanoliquid past a stretchable sheet. One of the most often addressed problems is heat transfer analysis in laminar flow past sheets/pipes/tubes. Both academic and industrial uses exist for laminar flows. Laminar flow devices are increasingly being used in the electronics and aerospace sectors to manage airborne particulate matter. Many laminar flows serve as standards for developing improved simulation methods. The laminar domain is typically formed in flows with low velocity, low density, or high viscosity from an industrial standpoint [31,32].

To the best of our knowledge, the effect of chemical reaction on the non-Newtonian liquid flow over a stretching sheet with a porous medium under LTNE conditions have not been explored in the literature. The present paper's main focus is to numerically examining the aforementioned flow. It is common knowledge that there are a variety of methods that may be considered in order to come up with viable solutions to this kind of issue. However, no numerical solution has yet been explored for the above-described flow. This research gap prompted us to utilize a numerical technique (the classical RK method) and a shooting scheme [33-37] to explore the effects of key factors on the flow features of Oldroyd-B and Jeffrey flow inside the interface. Finally, this paper's goal was to provide solutions to the following related research questions:

* What effects do several significant factors have on the flow, mass, and heat transfer behaviour of Oldroyd-B and Jeffrey liquids?

* What effects do the increase of different dimensionless variables have on the solid and liquid phase heat transfer rates?

* What effects do the increase of different dimensionless variables have on skin friction and mass transfer rate?

\section{Mathematical Formulation}

Consider a steady, incompressible, laminar flow of non-Newtonian liquid (Oldroyd-B and Jeffrey) over a sheet in a two-dimensional cartesian coordinate system $(x, y)$. With velocity components $\mathrm{u}, \mathrm{v}$ in $(x, y)$ directions, the $x$-axis is taken in the direction of the main flow over the plate, and the $y$-axis is normal to the plate. Further, it is assumed that the sheet is being stretched with the velocity $U_{w}=c x$ in a porous medium-a characteristic Darcy model. The flow is restricted to $y>0$, and coincides with the plane $y=0$, as shown in Figure 1. Each boundary wall is assumed to be impermeable and with perfect thermal conductance. Furthermore, the mass transfer process is considered in the occurrence of chemical reaction. Homogeneousness in the porous medium is assumed. The heat transfer analysis of the liquid and solid phases is analysed by using two different heat transport equations [1,4]. It is also worth noting that these equations are in the form of time-invariant partial differential equations. 


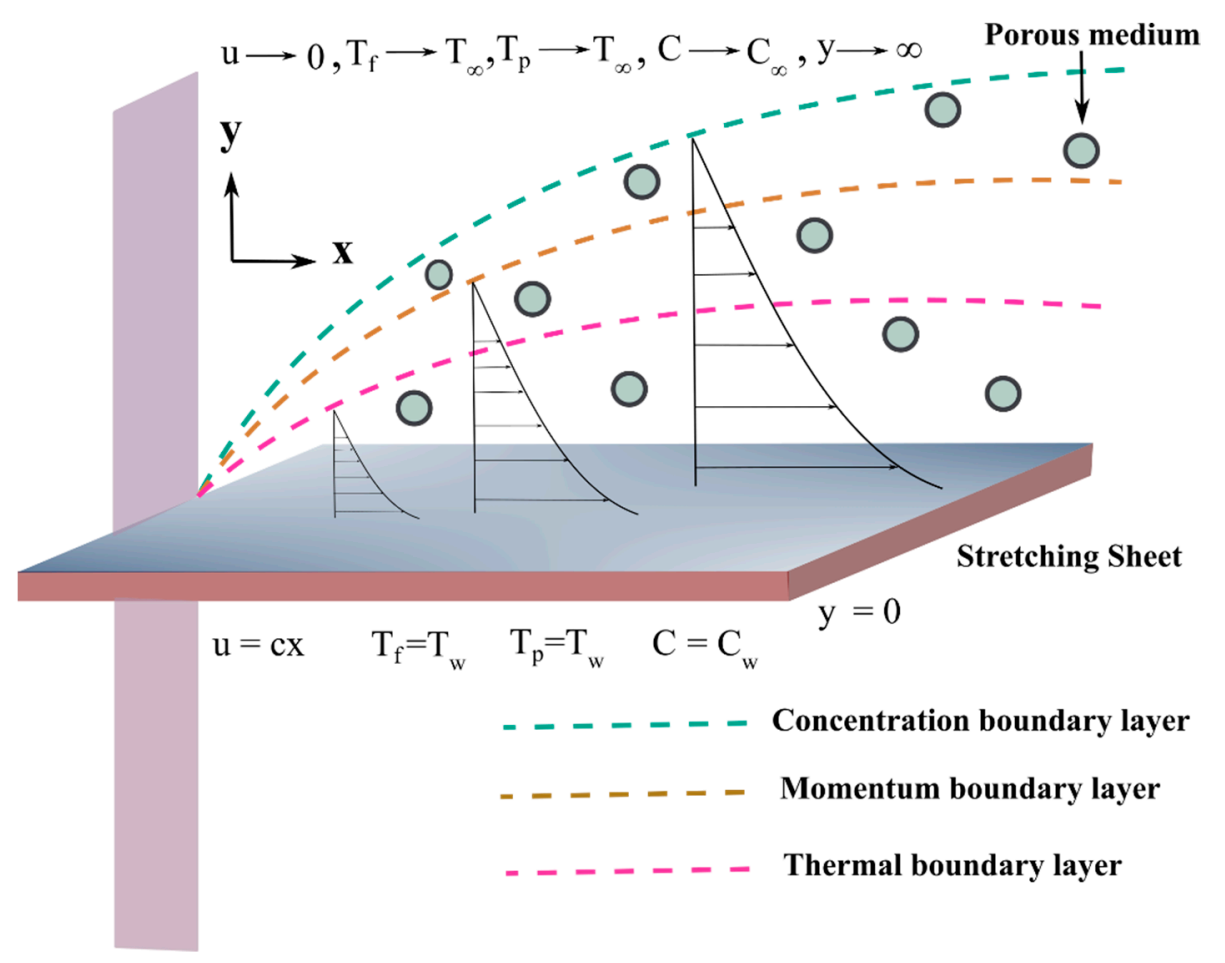

Figure 1. Flow geometry.

The equations for the assumed flow, heat, and mass transfer scrutiny inside the porous medium takes the following form [4,16,17]:

Continuity equation:

$$
\frac{\partial u}{\partial x}+\frac{\partial v}{\partial y}=0
$$

The continuity equation, Equation (1), states that inflow is equal to outflow. Here, $(u, v)\left[\mathrm{ms}^{-1}\right]$ are velocity components in the $(x, y)[\mathrm{m}]$ directions.

Momentum equation:

$$
\begin{aligned}
& \frac{1}{\varepsilon^{2}}\left[u \frac{\partial u}{\partial x}+v \frac{\partial u}{\partial y}\right]+\beta_{1}\left[u^{2} \frac{\partial^{2} u}{\partial x^{2}}+v^{2} \frac{\partial^{2} u}{\partial y^{2}}+2 u v \frac{\partial^{2} u}{\partial x \partial y}\right]= \\
& \frac{\mu_{f}}{\rho_{f}\left(1+\beta_{2}\right)}\left(\frac{\partial^{2} u}{\partial y^{2}}+\beta_{3}\left(u \frac{\partial^{3} u}{\partial x \partial y^{2}}+v \frac{\partial^{3} u}{\partial y^{3}}-\frac{\partial u}{\partial x} \frac{\partial^{2} u}{\partial y^{2}}+\frac{\partial u}{\partial y} \frac{\partial^{2} u}{\partial x \partial y}\right)\right)-\frac{\mu_{f}}{\rho_{f}} \frac{u}{K^{*}} .
\end{aligned}
$$

Equation (2) is a momentum equation that holds the momentum conservation rule. Inertial forces are responsible for the first term on the left hand side of Equation (2), while the last term is due to the additional effect of the porous medium. The relaxation time-or the time it takes for a reaction to achieve equilibrium - is represented by the parameter $\beta_{1}[\mathrm{~s}]$. In general, there are many relaxation times, each of which is linked to an internal reaction. However, a black box approach and a single relaxation time are used for the whole system ([38]). $\varepsilon$ is the dimensionless porosity. The parameter $\beta_{2}$ is the relaxation and retardation time ratio. The parameter $\beta_{3}[\mathrm{~s}]$ indicates the retardation time, which is the period between the application of a force and the manifestation of its effect by [39]. Because viscoelastic liquids do not react instantly to a rapid change in stress, they have a retardation time ([39]). Here, $\mu\left[\mathrm{kgm}^{-1} \mathrm{~s}^{-1}\right]$ is the dynamic viscosity, $\rho\left[\mathrm{kgm}^{-3}\right]$ is the density, and $K^{*}\left[\mathrm{~m}^{2}\right]$ is the porous medium's permeability. 
Energy equations for fluid and solid phases:

$$
\begin{gathered}
\frac{1}{\varepsilon}\left[u \frac{\partial T_{f}}{\partial x}+v \frac{\partial T_{f}}{\partial y}\right]=\frac{k_{f}}{\left(\rho C_{p}\right)_{f}}\left(\frac{\partial^{2} T_{f}}{\partial y^{2}}+\frac{\partial^{2} T_{f}}{\partial x^{2}}\right)+\frac{h_{f s}}{\varepsilon\left(\rho C_{p}\right)_{f}}\left(T_{s}-T_{f}\right), \\
0=\frac{k_{s}}{\left(\rho C_{p}\right)_{s}}\left(\frac{\partial^{2} T_{s}}{\partial y^{2}}+\frac{\partial^{2} T_{s}}{\partial x^{2}}\right)+\frac{h_{f s}}{(1-\varepsilon)\left(\rho C_{p}\right)_{s}}\left(T_{f}-T_{s}\right) .
\end{gathered}
$$

Equation (3) states that heat may be transferred through a fluid via convection (LHS), by conduction (first term on RHS), or by heat exchanges between the fluid and solid-matrix phases (last terms on RHS).

The nature of the saturating fluid and porous matrix determine the interphase heat transfer coefficient $h$, and the value of this coefficient has been the topic of great experimental attention. Small values of $h$ give rise to relatively significant thermal non-equilibrium effects, whereas large values of $h$ correspond to a fast movement of heat between the phases (LTE) [40].

Diffusion equation:

$$
\frac{1}{\varepsilon}\left[u \frac{\partial C}{\partial x}+v \frac{\partial C}{\partial y}\right]=D\left(\frac{\partial^{2} C}{\partial y^{2}}+\frac{\partial^{2} C}{\partial x^{2}}\right)-k_{r}\left(C-C_{\infty}\right) .
$$

Equation (5) represents the diffusion equation or mass transfer equation. The last term of the RHS represents the additional effects of chemical reaction.

The pertinent boundary conditions for the velocity field are:

$$
\left.\begin{array}{c}
u=c x, v=0 \text { at } y=0 \\
u \rightarrow 0, \frac{\partial u}{\partial y} \rightarrow 0 \text { at } y \rightarrow \infty
\end{array}\right\}
$$

For the energy Equations (3) and (4), the thermal boundary conditions are:

$$
\left.\begin{array}{c}
T_{f}=T_{w}, T_{p}=T_{w} \text { at } y=0 \\
T_{f} \rightarrow T_{\infty}, T_{p} \rightarrow T_{\infty} \text { as } y \rightarrow \infty .
\end{array}\right\}
$$

The boundary conditions for the diffusion Equation (5) are:

$$
\left.\begin{array}{c}
C=C_{w} \text { at } y=0 \\
C \rightarrow C_{\infty} \text { as } y \rightarrow \infty
\end{array}\right\}
$$

where $c\left[\mathrm{~s}^{-1}\right]$ is the stretching constant, $T[\mathrm{~K}]$ is the temperature, $C$ is the concentration, $D\left[\mathrm{~m}^{2} \mathrm{~s}^{-1}\right]$ is the diffusivity, $k_{r}\left[\mathrm{~s}^{-1}\right]$ is the reaction rate. $h_{f s}\left[\mathrm{kgs}^{-3} \mathrm{~m}^{-1} \mathrm{~K}^{-1}\right]$ is the heat transfer coefficient for the fluid-solid interface, $k\left[\mathrm{kgms}^{-3} \mathrm{~K}^{-1}\right]$ is the thermal conductivity, and $T_{\infty}$ and $C_{\infty}$ represent the ambient temperature and ambient concentration of the fluid, respectively. Similarly, $T_{w}$ and $C_{w}$ are the wall temperature and wall concentration of the fluid, respectively. The suffix $f$ represents the base fluid phase, while $s$ represents the solid-matrix phase.

The proposed mathematical model is deliberated based on the following conditions:

i. $\quad$ The problem represents Jeffrey fluid, if $\beta_{1}=0, \beta_{2} \neq 0, \beta_{3} \neq 0$;

ii. The problem represents Oldroyd-B liquid, if $\beta_{1} \neq 0, \beta_{2}=0, \beta_{3} \neq 0$.

\subsection{Similarity Transformations Used in the Model}

The experimenters must discover similarity transformations that decrease the number of independent variables in the problem in order to collapse measurements or calculations performed in a three-dimensional space into a two-dimensional plot. The use of similarity transformations aims at reducing the complexity of the problem. The similarity transfor- 
mations method involves converting partial differential equations to ordinary differential equations that may be solved in a traditional manner. The similarity transformations used in the modelling are as follows:

$$
\begin{aligned}
& \psi=\sqrt{c v_{f}} x f(\eta), u=\frac{\partial \psi}{\partial y}=c x f^{\prime}(\eta), v=-\frac{\partial \psi}{\partial x}=-\sqrt{c v_{f}} f(\eta), \eta=\sqrt{\frac{c}{v_{f}}} y, \\
& \theta_{f}(\eta)=\frac{T_{f}-T_{\infty}}{T_{w}-T_{\infty}}, \theta_{s}(\eta)=\frac{T_{s}-T_{\infty}}{T_{w}-T_{\infty}}, \chi(\eta)=\frac{c-C_{\infty}}{C_{w}-C_{\infty}} .
\end{aligned}
$$

where, $\theta_{\mathcal{S}}(\eta)$ represents the solid-phase thermal profile, $\theta_{f}(\eta)$ represents the fluid-phase temperature profile, and $\chi(\eta)$ is the concentration profile. One of the most challenging aspects of calculating the flow of an incompressible liquid is that the equations of momentum must be solved under the continuity constraint, regardless of their form (Equation (2)). The vorticity/stream function technique is a well-known method of solving challenges in two-dimensional flow issues. The equation of continuity is fulfilled by expressing velocity in terms of a stream function $\psi$, which guarantees that the velocity field is solenoidal. Thus, one governing equation (the continuity Equation (2)) is eliminated.

Furthermore, by using Equation (7), the momentum Equation (2) is reduced as follows:

$$
\begin{aligned}
& f^{\prime \prime \prime}+\lambda_{2}\left[f^{\prime \prime}{ }^{2}-f f^{i v}\right]-\frac{\left(1+\beta_{2}\right)}{\varepsilon^{2}}\left[f^{\prime 2}-f f^{\prime \prime}\right]-\left(1+\beta_{2}\right) K^{* *} f^{\prime} \\
& -\left(1+\beta_{2}\right)\left[\lambda_{1}\left(f^{2} f^{\prime \prime \prime}-2 f f^{\prime} f^{\prime \prime}\right)\right]=0 .
\end{aligned}
$$

The reduced boundary conditions of the velocity field become:

$$
f^{\prime}(0)=1, f(0)=0, f^{\prime}(\infty) \rightarrow 0, f^{\prime \prime}(\infty) \rightarrow 0 .
$$

where the non-dimensional parameters in the momentum equation are as follows:

$\lambda_{2}=\beta_{3} c$ is the Deborah number with respect to retardation time, $K^{* *}=\frac{v_{f}}{K^{*} c}$ is the porosity parameter, and $\lambda_{1}=\beta_{1} c$ is the Deborah number with respect to relaxation time.

The energy Equations (3) and (4) can be reduced to the following forms:

$$
\begin{gathered}
\frac{1}{\operatorname{Pr}} \theta_{f}^{\prime \prime}+\frac{1}{\varepsilon} f \theta_{f}^{\prime}+H\left(\theta_{s}-\theta_{f}\right)=0, \\
\frac{1}{\operatorname{Pr}} \theta_{s}^{\prime \prime}+\gamma H\left(\theta_{f}-\theta_{s}\right)=0 .
\end{gathered}
$$

The corresponding reduced thermal boundary conditions for the energy equations are:

$$
\theta_{f}(0)=1, \theta_{s}(0)=1, \theta_{f}(\infty) \rightarrow 0, \theta_{s}(\infty) \rightarrow 0 .
$$

where the non-dimensional parameters in energy equations are as follows:

$\gamma=\frac{\varepsilon k_{f}}{(1-\varepsilon) k_{s}}$ is the porosity-modified conductivity ratio, $\operatorname{Pr}=\frac{v_{f}}{\alpha_{f}}$ is the Prandtl numberm and $H=\frac{h_{f s} \alpha_{f}}{\varepsilon c k_{f}}$ is the non-dimensional interphase heat transfer parameter.

The reduced diffusion equation is as follows:

$$
\frac{1}{S c} \chi^{\prime \prime}+\frac{1}{\varepsilon} f \chi^{\prime}-\sigma \chi=0
$$

along with the reduced boundary conditions for the diffusion equation:

$$
\chi(0)=1, \chi(\infty) \rightarrow 0 .
$$

where the non-dimensional parameters in the diffusion equation are as follows:

$S c=\frac{v_{f}}{D}$ is the Schmidt number, while $\sigma=\frac{k_{r}}{c}$ is the chemical reaction rate parameter. 


\subsection{The Quantities for Engineering Interest}

One of the most significant variables in aeronautical engineering is wall shear stress, often known as skin friction; this is responsible for a significant part of the drag on commercial aircraft, and is widely utilised by the CFD community to determine the velocity profile of the boundary layer. The local skin friction $\left(C_{f}\right)$ parameter is used to determine the resistance to the flow. The relation in its non-dimensional form is as follows:

$$
\sqrt{\operatorname{Re}} C_{f}=2\left(\frac{1+\lambda_{1}}{1+\lambda_{2}}\right) f^{\prime \prime}(0) .
$$

The Nusselt number $(\mathrm{Nu})$ is the convective-to-conductive heat transport ratio at a fluid's boundary. Advection (fluid motion) and diffusion are both types of convection (conduction). Nusselt number relations are used to determine the rate of heat transfer; these relations in their dimensionless forms take the following structure for fluid and solid phases, respectively:

$$
\begin{aligned}
& \frac{N u_{f}}{\sqrt{\operatorname{Re}}}=-\theta^{\prime}{ }_{f}(0), \\
& \frac{N u_{s}}{\sqrt{\operatorname{Re}}}=-\theta^{\prime}{ }_{s}(0),
\end{aligned}
$$

where $N u_{f}$ and $N u_{s}$ represent the Nusselt numbers for the liquid and solid phases, respectively.

The Sherwood number $(S h)$ is a non-dimensional number used in mass transference operations. It is also known as the mass transfer Nusselt number. It is the proportion of convective mass transfer to diffusive mass transport rate. This relation in its nondimensional form is as follows:

$$
\frac{S h}{\sqrt{\mathrm{Re}}}=-\chi^{\prime}(0),
$$

where Re is the local Reynolds number and $S h$ is the Sherwood number.

\section{Numerical Procedure}

Not all boundary value problems can be solved analytically, but the solution to a well-defined initial value problem is easy to provide. This leads to numerical solutions, which consist of the shooting method and Runge-Kutta integration scheme. These methods have gained popular significance due to their applications in resolving problems in science and engineering. It is very important to choose the appropriate integrator, as this decides the accurateness of the shooting process. The system of Equations (10), (12), (13) and (15) with the boundary constraints (11), (14), and (16) have been numerically solved by applying the Runge-Kutta integration scheme together with the shooting iteration procedure. Thus, Equations (10)-(16) are transformed into a system of first-order differential equations.

Later, the shooting method is used to guess the missing initial conditions via iterative process, and undetermined initial conditions are presumed. Then, the resultant conditions are integrated with the help of the Runge-Kutta process. Iteratively comparing the estimated value of the dependent variable at the terminal point with its supplied value improves the values of presumed missing initial conditions. The value of $\eta$ is chosen sufficiently large so that the boundary conditions are asymptotically satisfied. A program was written using MATLAB to solve the problem. A step size of $\Delta \eta=0.0001$ was selected to be satisfactory for a convergence criterion of $10^{-6}$ in nearly all cases. The numerical findings are also compared for the thermal gradient obtained with existing works shown in Table 1. Here, the obtained numerical solutions are compared to solutions obtained by Ishak et al. [26] (finite-element method), Vajravelu et al. [27] (Keller box method), and Prakash et al. [4] (Runge-Kutta-Fehlberg method), who all used different methods to solve models related to boundary value flow past stretching surfaces, and attained a better agreement with them. 
Table 1. Comparison of results for the thermal gradient with published papers for some reduced cases.

\begin{tabular}{|c|c|c|c|c|c|c|}
\hline \multirow{3}{*}{ Pr } & \multicolumn{4}{|c|}{ Published Papers $\theta^{\prime}(0)$} & \multicolumn{2}{|c|}{ Present Results } \\
\hline & \multirow{2}{*}{ Ishak et al. [26] } & \multirow{2}{*}{ Vajravelu et al. [27] } & \multicolumn{2}{|c|}{ Prakash et al. [4] } & \multirow[b]{2}{*}{$\theta_{s}^{\prime}(0)$} & \multirow{2}{*}{$\boldsymbol{\theta}_{f}^{\prime}(\mathbf{0})$} \\
\hline & & & $\theta_{s}^{\prime}(0)$ & $\theta_{f}^{\prime}(0)$ & & \\
\hline 0.7 & 0.8086 & 0.808836 & 0.808570 & 0.808578 & 0.808619 & 0.808625 \\
\hline 1 & 1.0000 & 1.000000 & 0.999927 & 0.999937 & 0.999945 & 0.999953 \\
\hline 2 & 1.9237 & 1.923687 & 1.923556 & 1.923573 & 1.923675 & 1.923685 \\
\hline 10 & 3.7207 & 3.720788 & 3.720444 & 3.720475 & 3.720616 & 3.720632 \\
\hline
\end{tabular}

\section{Results and Discussion}

The effect of chemical reaction on the non-Newtonian liquid stream on an SS with a porous medium under LTNE circumstances is explored in this study. This section includes a detailed explanation, as well as the impact of key relevant factors on the corresponding distributions. The influence of physical parameters on these features is illustrated one by one graphically. All computations are made for a wide range of flow issue parameters. The impacts of non-dimensional parameters on the involved profiles of Jeffrey and Oldroyd-B liquids are graphically analysed. Furthermore, a comparative investigation of Jeffrey and Oldroyd B liquids is conducted. For all of these, the Prandtl number was fixed to 20.1, while other variables were set to vary in order to analyse the effects on the flow, mass and heat transport.

Figure 2a shows the impact of $K^{* *}$ on $f^{\prime}(\eta)$ for both Oldroyd-B and Jeffrey liquids. The increase in $K^{* *}$ reduces the $f^{\prime}(\eta)$ of both liquids. The resistance of the system increases as the porosity component value increases. Because of the increased frictional force, fluid flow is reduced. Increased porosity enhances the surface's barrier to liquid mobility in this scenario. The additional resistance slows down the liquid's velocity. Furthermore, at lower $K^{* *}$ values, $f^{\prime}(\eta)$ for Oldroyd-B liquid is strongly stimulated, and decreases quicker than Jeffrey liquid. However, at higher $K^{* *}$ values, the $f^{\prime}(\eta)$ of Jeffery liquid is severely stimulated, and decreases quicker than the $f^{\prime}(\eta)$ of Oldroyd-B liquid. Figure $2 \mathrm{~b}$ shows the dominance of $K^{* *}$ over $\theta_{f}(\eta)$ for both liquids. The increase in $K^{* *}$ raises the $\theta_{f}(\eta)$ of both liquids. The fluids become more viscous as $K^{* *}$ grows, and the fluid's velocity stagnates as a result of the increased viscosity, which inhibits fluid mobility across the surface. It is evident that the presence of a permeable substance restricts the flow of liquid, resulting in a reduction in liquid velocity and an upsurge in heat transfer. Furthermore, Oldroyd-B liquid has better heat transference than Jeffrey liquid for lower porosity values, whereas Jeffrey liquid has better heat transfer than Oldroyd-B liquid at higher porosity levels. Figure 3 a demonstrates the power of $\gamma$ over the $\theta_{f}(\eta)$ of both fluids. The increasing values of $\gamma$ cause a decrease in the $\theta_{f}(\eta)$ for both Oldroyd-B and Jeffrey liquids. According to the definition of the $\gamma$, the rising values of $\gamma$ have the ability to decrease the fluid-phase heat transfer. Moreover, the fluid-phase heat transfer of Jeffrey liquid is greater than that of Oldroyd-B liquid, and decays slowly for growing values of $\gamma$. Figure $3 \mathrm{~b}$ exhibits the effects of $\gamma$ on the $\theta_{S}(\eta)$ of both liquids. The growth in $\gamma$ causes a decrease in the $\theta_{s}(\eta)$ of both liquids. Increasing $\gamma$ causes the system to become unstable. The influence is most obvious when there is very little $\gamma$. Convection may be totally suppressed at higher $\gamma$ values, leading to the declination of both fluid- and solid-phase temperature profiles. Furthermore, the solid-phase heat transfer of Jeffrey liquid is greater than that of Oldroyd-B liquid, and decays slowly as $\gamma$ increases. 

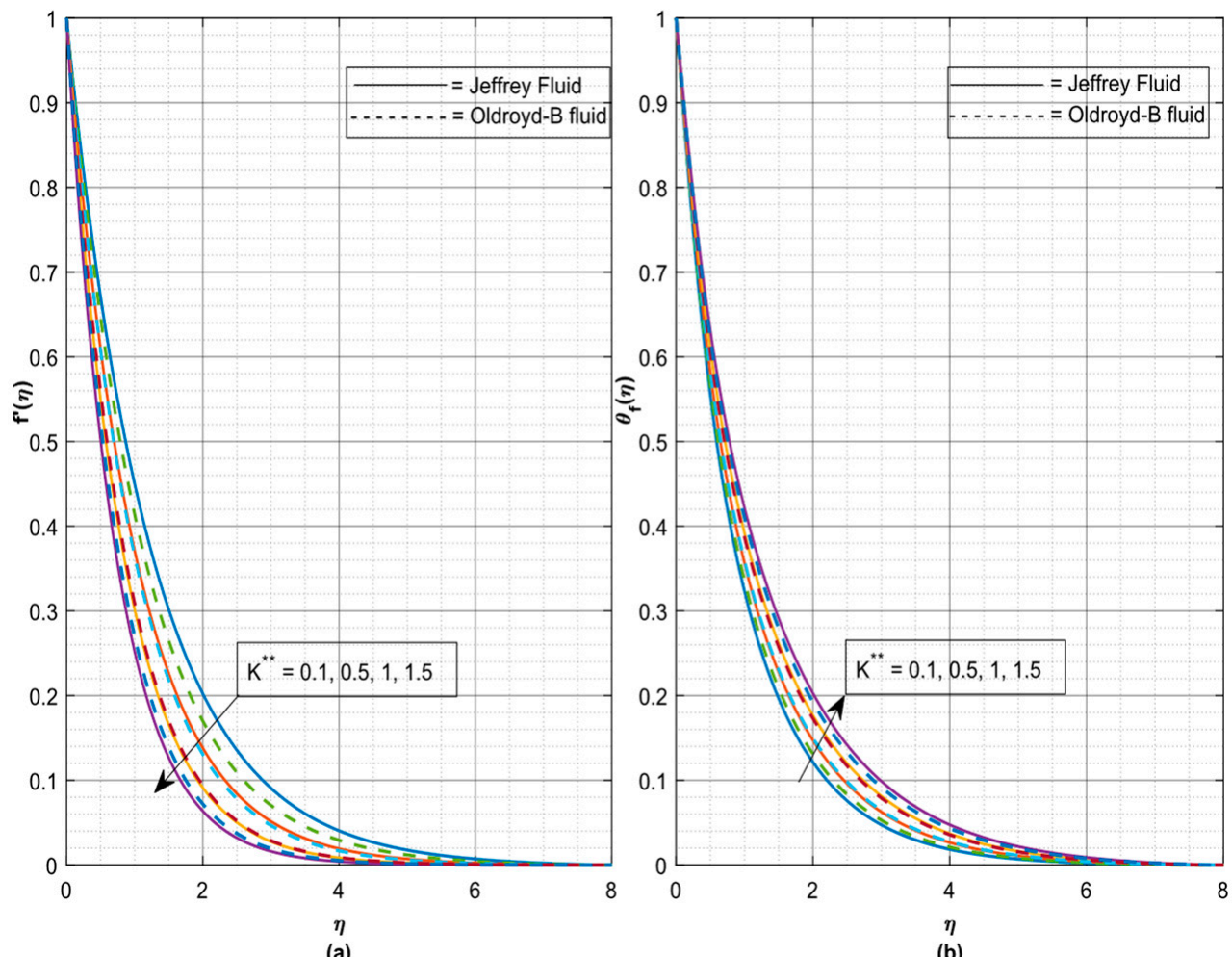

Figure 2. (a) Effect of $K^{* *}$ on $f^{\prime}(\eta)$, and (b) effect of $K^{* *}$ on $\theta_{f}(\eta)$.
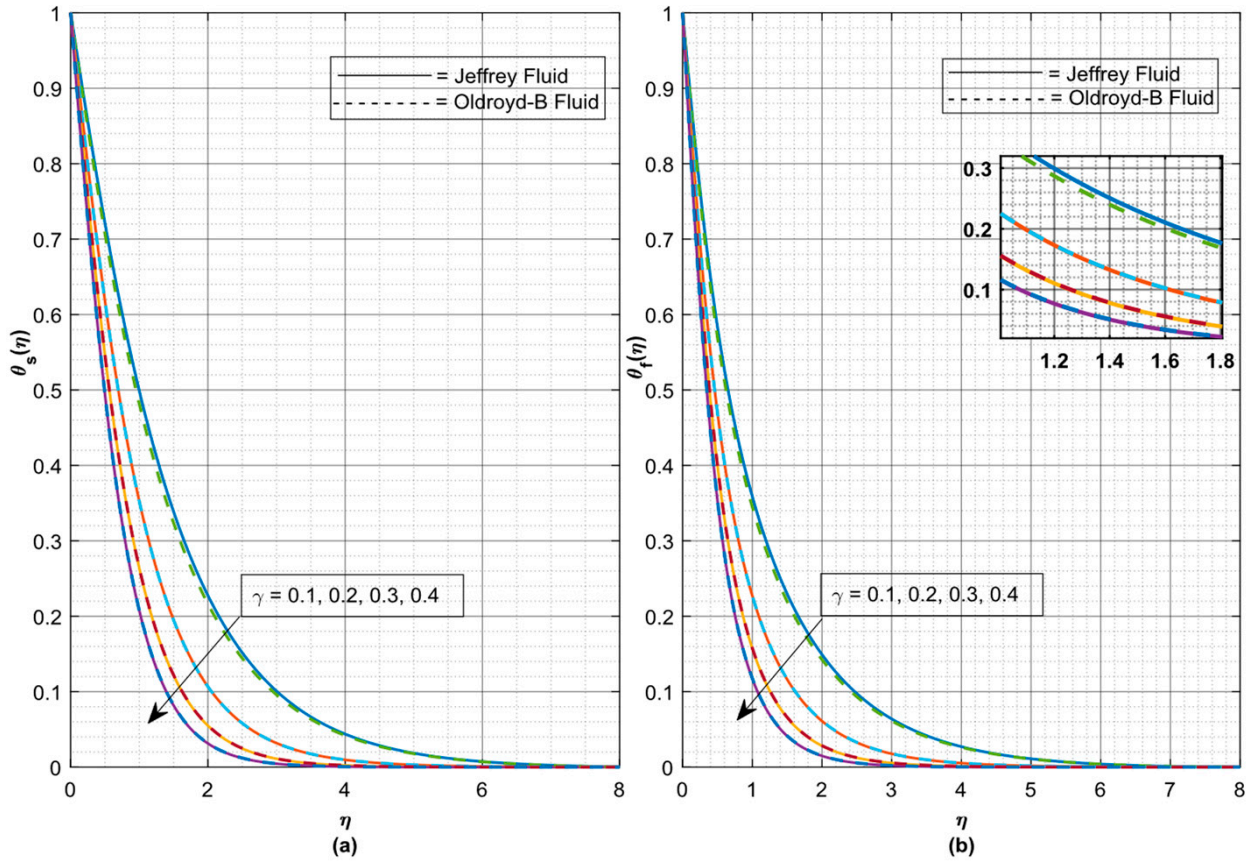

Figure 3. (a) Effect of $\gamma$ on $\theta_{f}(\eta)$, and (b) effect of $\gamma$ on $\theta_{\mathcal{S}}(\eta)$. 
Figure 4a illustrates the effects of $H$ on the $\theta_{f}(\eta)$ of both liquids. The growth in $H$ causes an increase in the $\theta_{f}(\eta)$ of both liquids. Furthermore, both liquids show approximately similar fluid-phase heat transfer for escalating values of $H$. Figure $4 \mathrm{~b}$ displays the effects of $H$ on the $\theta_{s}(\eta)$ of both Oldroyd-B and Jeffrey liquids. The growth in $H$ causes a decrease in the $\theta_{s}(\eta)$ of both liquids. Furthermore, at increasing values of $H$, both liquids exhibit almost the same solid-phase heat transfer. As $H$ rises, the thermal gradient of the solid phase decreases, while that of the fluid phase rises, owing to quick heat transfer between the phases. Figure 5a shows the effects of $\sigma$ on the $\chi(\eta)$ of both Oldroyd-B and Jeffrey liquids. The increase in $\sigma$ causes a decrease in the $\chi(\eta)$ of both liquids. The distribution of solutal concentration becomes weaker in the event of a chemical reaction. As a consequence, as the chemical reaction parameter grows, the solutal concentration boundary layer becomes thinner. Chemical reactions speed up the rate of interfacial mass transfer. Further, Jeffrey liquid shows improved fluid-phase mass transfer and decays more slowly compared to Oldroyd-B fluid for higher $\sigma$ values. The impact of $S c$ on the $\chi(\eta)$ of both fluids is disclosed in Figure 5b. The increase in $S c$ causes a decrease in the $\chi(\eta)$. The growing value of $S c$ increases momentum diffusivity and causes the mass transport to deteriorate. The Schmidt number is a dimensionless number that describes the relationship between mass and momentum diffusivities in a fluid flow. The smallest $S c$ refers to the highest concentration of the nanoparticles. The higher values of $S c$ cause a decrease in the diffusion coefficient, which slows down the mass transfer. Furthermore, the $\chi(\eta)$ for Jeffrey liquid decays more slowly than that of the Oldroyd-B liquid for escalating values of Sc.
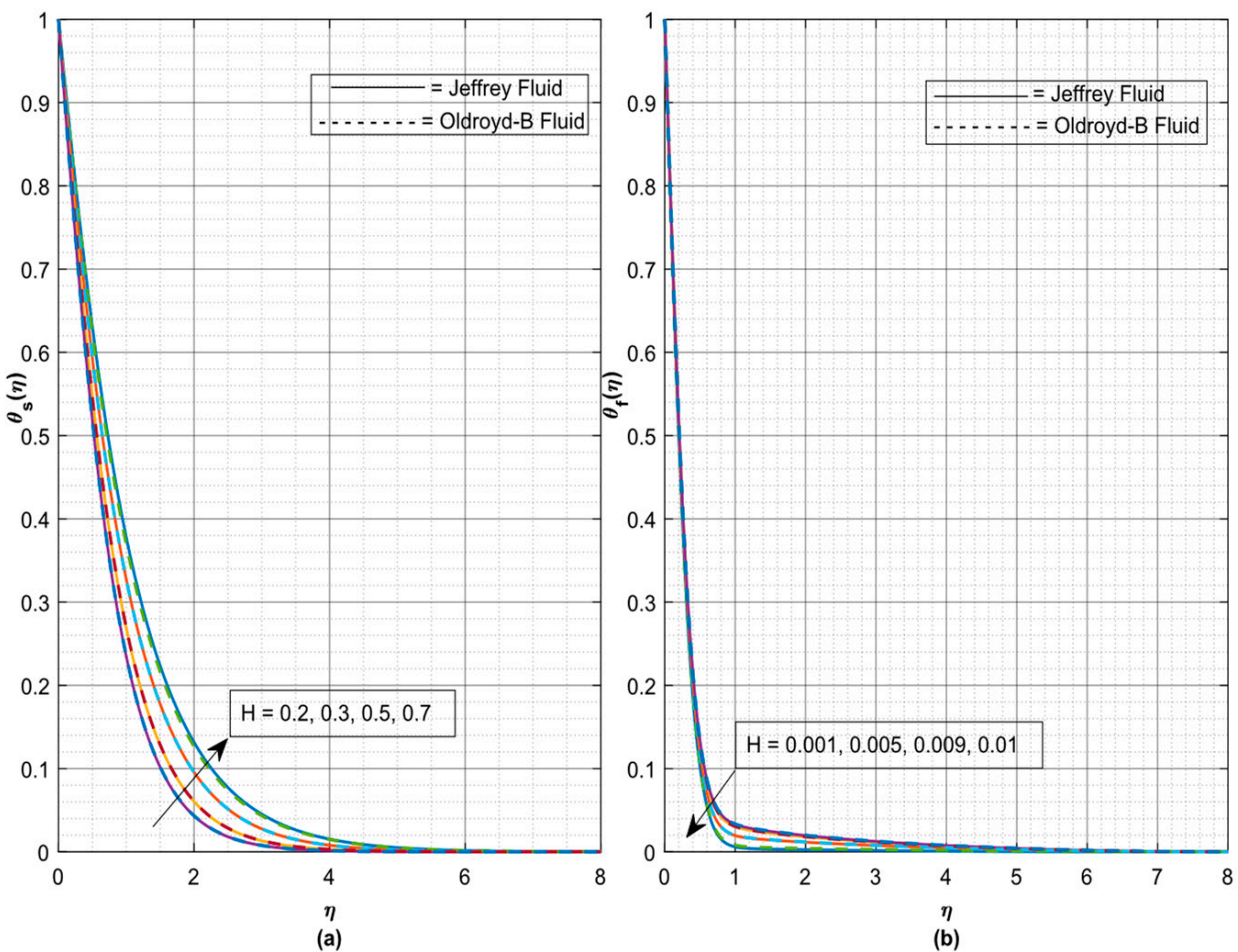

Figure 4. (a) Effect of $H$ on $\theta_{f}(\eta)$, and (b) effect of $H$ on $\theta_{S}(\eta)$. 


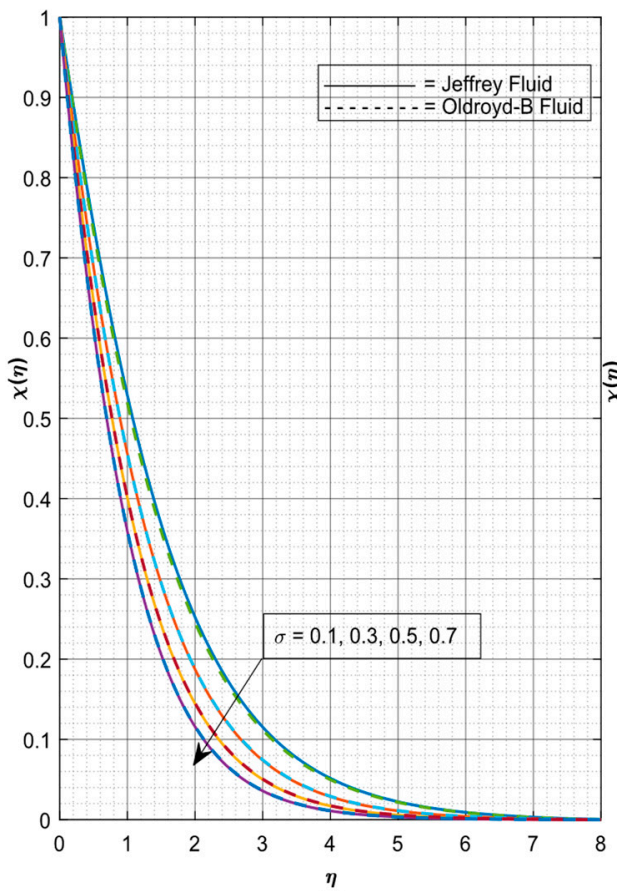

(a)

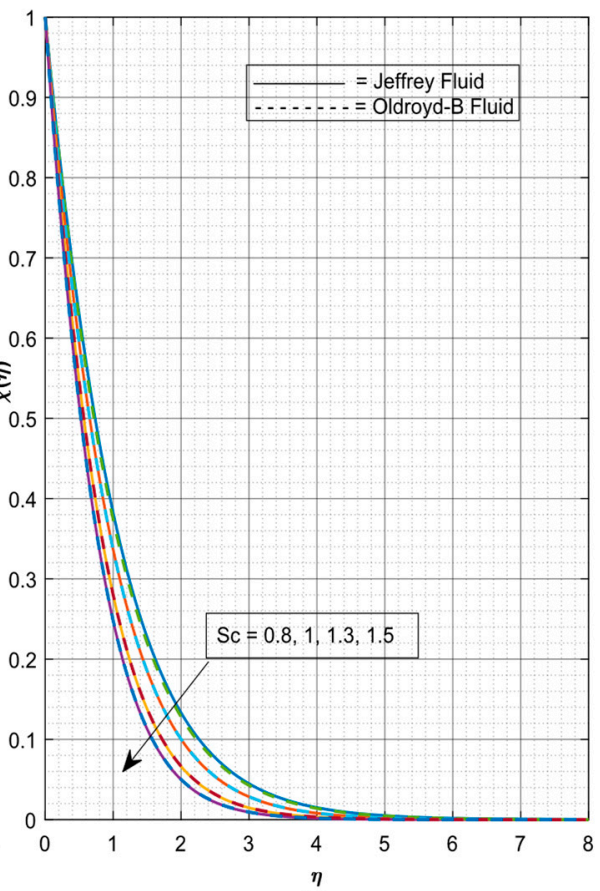

(b)

Figure 5. (a) Effect of $\sigma$ on $\chi(\eta)$, and (b) effect of $S c$ on $\chi(\eta)$.

Figure 6a depicts the effects of $\lambda_{2}$ and $K^{* *}$ on the $\operatorname{Re}^{1 / 2} C_{f}$ of both fluids. The escalating values of $\lambda_{2}$ cause an increase in the skin friction, but improved values of $K^{* *}$ cause a decrease in the $\operatorname{Re}^{1 / 2} C_{f}$ of both liquids. Moreover, the $\operatorname{Re}^{1 / 2} C_{f}$ for Jeffrey liquid declines faster than that of the Oldroyd-B liquid. The fluctuation in $S h R e^{-1 / 2}$ versus $S c$ for escalating values of $\sigma$ for both liquids is revealed in Figure 6b. Here, $S h R e^{-1 / 2}$ improves with larger values of both $\sigma$ and Sc. Moreover, Jeffrey liquid shows a lower mass transfer rate than that of the Oldroyd-B liquid with increasing values of both $\sigma$ and $S_{c}$. The effects of $H$ on the $\operatorname{Re}^{-1 / 2} N u_{f}$ versus $\gamma$ of both liquids are typified in Figure 7a. The intensifying values of $H$ cause a decrease in the $\mathrm{Re}^{-1 / 2} \mathrm{Nu}$ of both liquids, but divergent movement is seen for improved $\gamma$ values. Furthermore, at increasing $H$ values, Oldroyd-B liquid has a slightly higher fluid-phase heat transfer rate than Jeffrey liquid. The effects of $K^{* *}$ on the $\operatorname{Re}^{-1 / 2} N u_{s}$ versus $\gamma$ of both liquids are exemplified in Figure $7 \mathrm{~b}$. The escalating values of $K^{* *}$ cause a decrease in the $\mathrm{Re}^{-1 / 2} N u_{s}$ of both fluids, but inverse movement is seen for improved values of $\gamma$. Moreover, Oldroyd-B liquid shows an improved solid-phase heat transfer rate compared to Jeffrey liquid for increasing values of both $K^{* *}$ and $\gamma$. 


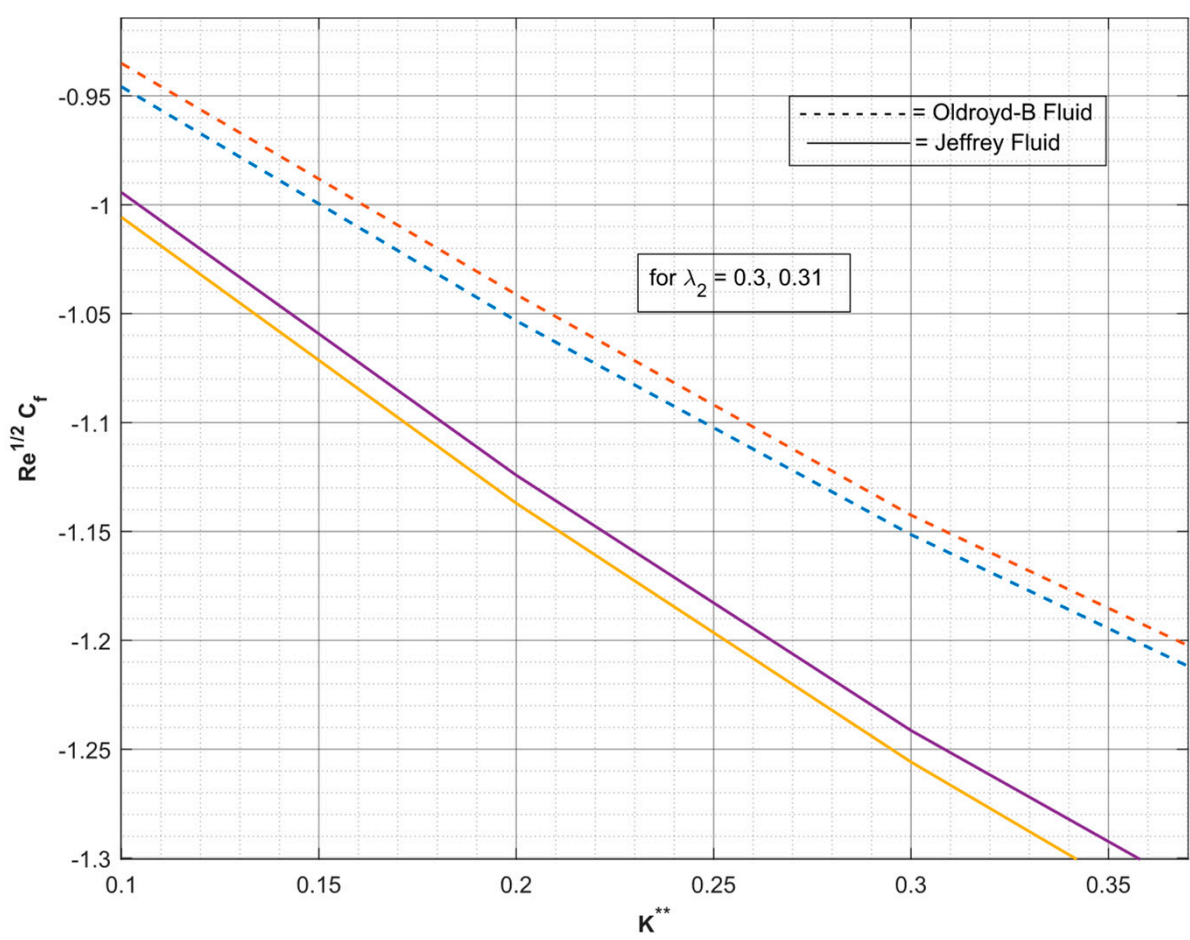

(a)

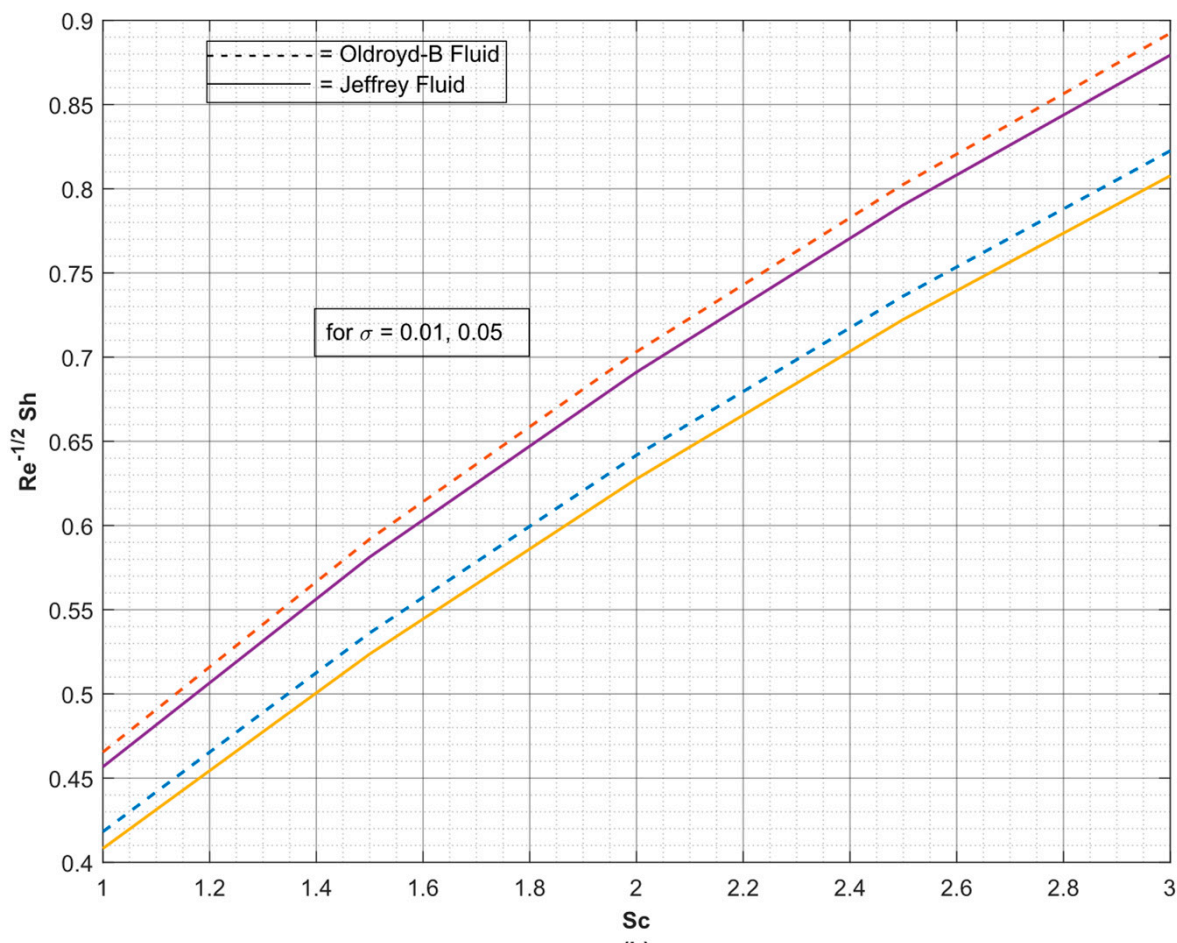

(b)

Figure 6. (a) Plot for skin friction, and (b) plot for Sherwood number. 


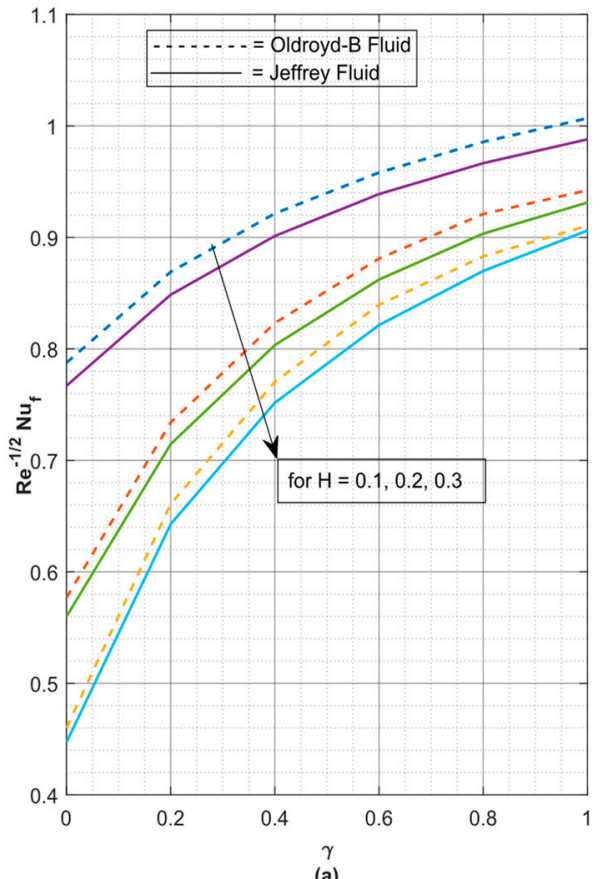

(a)

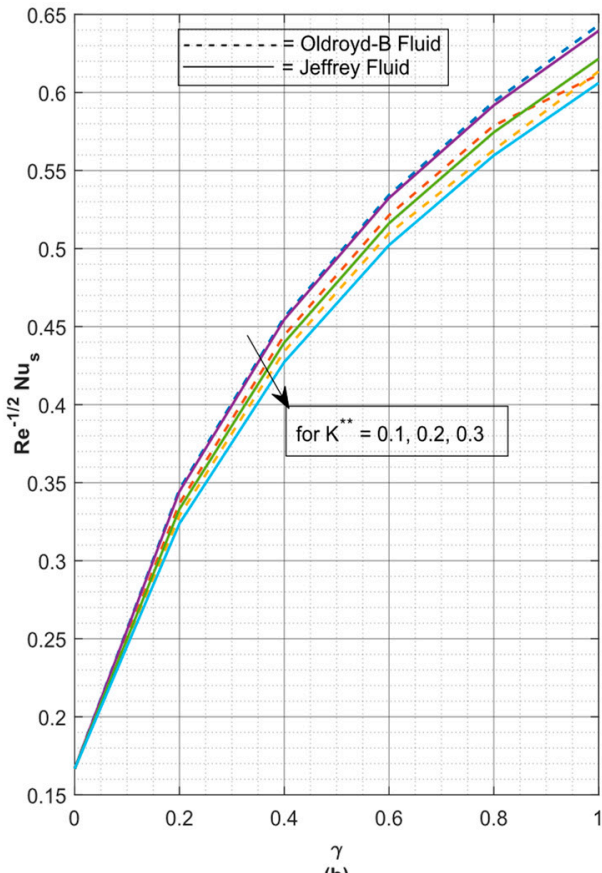

(b)

Figure 7. (a) Plot for fluid phase Nusselt number, and (b) plot for solid phase Nusselt number.

\section{Conclusions}

The current study explores the effects of chemical reaction on the flow of nonNewtonian liquid on a stretching sheet with a porous medium under LTNE conditions. The model equation system is reduced by using appropriate similarity transformations, which are then solved numerically by employing the shooting method and the classical RK method. The influence of pertinent parameters on the involved profiles is analysed graphically. The main conclusions drawn from the current study are as follows:

- The increasing values of $K^{* *}$ cause an increase in the $f^{\prime}(\eta)$ of both liquids, but converse behaviour is seen in the $\theta_{f}(\eta)$ of both liquids;

- The liquid- and solid-phase heat transfer of Jeffrey liquids are more than those of Oldroyd-B liquid for increasing values of $\gamma$;

- The thermal gradient of the solid phase of both liquids drops, while the liquid phase of both liquids increases, as $H$ increases;

- Jeffrey liquid shows improved fluid phase-mass transfer and decays more slowly than Oldroyd-B liquid for higher values of both $\sigma$ and $S c$;

- The rising values of $H$ cause a decrease in the $\mathrm{Re}^{-1 / 2} \mathrm{~N} u_{f}$ of both liquids, but divergent movement is seen for improved $\gamma$ values;

- The escalating values of $K^{* *}$ cause a decrease in the $\operatorname{Re}^{-1 / 2} N u_{s}$ of both fluids, but inverse movement is seen for improved values of $\gamma$.

Author Contributions: Conceptualization, A.A. and B.M.P.; methodology, R.P.K.C., K.S. and B.M.P.; software, A.A.; validation, R.P.K.C., B.M.P., H.A.H.A. and A.A.; formal analysis, R.P.K.C. and A.A.; investigation, A.A.; resources, A.A.; writing-original draft preparation, B.M.P.; writing-review and editing, R.P.K.C., K.S. and H.A.H.A.; visualization, H.A.H.A.; supervision, A.A. All authors have read and agreed to the published version of the manuscript.

Funding: Taif University Researchers Supporting Project Number (TURSP-2020/47), Taif University, Taif, Saudi Arabia.

Institutional Review Board Statement: Not applicable.

Informed Consent Statement: Not applicable.

Data Availability Statement: Data are included within the manuscript. 
Acknowledgments: The authors would like to thank the Ministry of Education in Saudi Arabia and the Taif University Researchers Supporting Project Number (TURSP-2020/47), Taif University, Taif, Saudi Arabia.

Conflicts of Interest: The authors declare no conflict of interest.

\section{References}

1. Nield, D.A.; Bejan, A. Convection in Porous Media; Springer: New York, NY, USA, 1999.

2. Kuznetsov, A.V.; Nield, D.A. The effect of local thermal nonequilibrium on the onset of convection in a porous medium layer saturated by a nanofluid: Brinkman model. J. Porous Media 2011, 14, 285-293. [CrossRef]

3. Sheremet, M.; Pop, I.; Nazar, R. Natural convection in a square cavity filled with a porous medium saturated with a nanofluid using the thermal nonequilibrium model with a Tiwari and Das nanofluid model. Int. J. Mech. Sci. 2015, 100, 312-321. [CrossRef]

4. Prakash, D.; Muthtamilselvan, M.; Niu, X.-D. Unsteady MHD non-Darcian flow over a vertical stretching plate em-bedded in a porous medium with thermal non-equilibrium model. Adv. Appl. Math. Mech. 2016, 8, 52-66. [CrossRef]

5. Mahajan, A.; Sharma, M.K. Effects of local thermal nonequilibrium on the onset of convection in a magnetic nanofluid layer. Heat Transf. Res. 2020, 51, 689-705. [CrossRef]

6. Muthtamilselvan, M.; Prakash, D.; Doh, D.-H. Effect of thermal non-equilibrium on transient hydromagnetic flow over a moving surface in a nanofluid saturated porous media. J. Mech. Sci. Technol. 2014, 28, 3709-3718. [CrossRef]

7. Kumar, R.S.V.; Gowda, R.J.P.; Radhika, M.; Prasannakumara, B.C. Two-phase flow of dusty fluid with suspended hybrid nanoparticles over a stretching cylinder with modified Fourier heat flux. SN Appl. Sci. 2021, 3, 1-9. [CrossRef]

8. Xiong, P.-Y.; Hamid, A.; Chu, Y.-M.; Khan, M.I.; Gowda, R.J.P.; Kumar, R.N.; Prasannakumara, B.C.; Qayyum, S. Dynamics of multiple solutions of Darcy-Forchheimer saturated flow of Cross nanofluid by a vertical thin needle point. Eur. Phys. J. Plus 2021, 136, 1-22. [CrossRef]

9. Sharma, M.K.; Mahajan, A. Onset of convection in a magnetic nanofluid-saturated porous medium under local thermal nonequilibrium conditions. Spéc. Top. Rev. Porous Media Int. J. 2021, 12, 59-77. [CrossRef]

10. Gowda, R.J.P.; Kumar, R.N.; Prasannakumara, B.C. Two-Phase Darcy-Forchheimer Flow of Dusty Hybrid Nanofluid with Viscous Dissipation Over a Cylinder. Int. J. Appl. Comput. Math. 2021, 7, 95. [CrossRef]

11. Song, Y.-Q.; Khan, M.I.; Qayyum, S.; Gowda, R.J.P.; Kumar, R.N.; Prasannakumara, B.C.; Elmasry, Y.; Chu, Y.-M. Physical impact of thermo-diffusion and diffusion-thermo on Marangoni convective flow of hybrid nanofluid $\left(\mathrm{MnZiFe}_{2} \mathrm{O}_{4}-\mathrm{NiZnFe}_{2} \mathrm{O}_{4}-\mathrm{H}_{2} \mathrm{O}\right)$ with nonlinear heat source/sink and radiative heat flux. Mod. Phys. Lett. B 2021, 35, 2141006. [CrossRef]

12. Khan, M.I.; Qayyum, S.; Shah, F.; Kumar, R.N.; Gowda, R.P.; Prasannakumara, B.; Chu, Y.-M.; Kadry, S. Marangoni convective flow of hybrid nanofluid ( $\mathrm{MnZnFe}_{2} \mathrm{O}_{4}-\mathrm{NiZnFe}_{2} \mathrm{O}_{4}-\mathrm{H}_{2} \mathrm{O}$ ) with Darcy Forchheimer medium. Ain Shams Eng. J. 2021. [CrossRef]

13. Gowda, R.P.; Kumar, R.N.; Jyothi, A.; Prasannakumara, B.; Sarris, I. Impact of Binary Chemical Reaction and Activation Energy on Heat and Mass Transfer of Marangoni Driven Boundary Layer Flow of a Non-Newtonian Nanofluid. Processes 2021, 9, 702. [CrossRef]

14. Kumar, R.S.V.; Dhananjaya, P.G.; Kumar, R.N.; Gowda, R.J.P.; Prasannakumara, B.C. Modeling and theoretical investigation on Casson nanofluid flow over a curved stretching surface with the influence of magnetic field and chemical reaction. Int. J. Comput. Methods Eng. Sci. Mech. 2021, 1-8. [CrossRef]

15. Gowda, R.J.P.; Kumar, R.N.; Jyothi, A.M.; Prasannakumara, B.C.; Nisar, K.S. KKL correlation for simulation of nanofluid flow over a stretching sheet considering magnetic dipole and chemical reaction. ZAMM J. Appl. Math. Mech./Z. Angew. Math. Mech. 2021, e202000372. [CrossRef]

16. Sandeep, N.; Kumar, B.R.; Kumar, M.J. A comparative study of convective heat and mass transfer in non-Newtonian nanofluid flow past a permeable stretching sheet. J. Mol. Liq. 2015, 212, 585-591. [CrossRef]

17. Sandeep, N.; Sulochana, C. Momentum and heat transfer behaviour of Jeffrey, Maxwell and Oldroyd-B nanofluids past a stretching surface with non-uniform heat source/sink. Ain Shams Eng. J. 2018, 9, 517-524. [CrossRef]

18. Mabood, F.; Bognár, G.; Shafiq, A. Impact of heat generation/absorption of magnetohydrodynamics Oldroyd-B fluid impinging on an inclined stretching sheet with radiation. Sci. Rep. 2020, 10, 17688. [CrossRef]

19. Ibrahim, W.; Gadisa, G. Finite element solution of nonlinear convective flow of Oldroyd-B fluid with Cattaneo-Christov heat flux model over nonlinear stretching sheet with heat generation or absorption. Propuls. Power Res. 2020, 9, 304-315. [CrossRef]

20. Khan, S.U.; Al-Khaled, K.; Bhatti, M. Bioconvection analysis for flow of Oldroyd-B nanofluid configured by a convectively heated surface with partial slip effects. Surf. Interfaces 2021, 23, 100982. [CrossRef]

21. Ali, B.; Hussain, S.; Nie, Y.; Hussein, A.K.; Habib, D. Finite element investigation of Dufour and Soret impacts on MHD rotating flow of Oldroyd-B nanofluid over a stretching sheet with double diffusion Cattaneo Christov heat flux model. Powder Technol. 2020, 377, 439-452. [CrossRef]

22. Pandikunta, S.; Malleswari, B.; Bhaskar Reddy, N. Electrical resistance heating distribution on three dimen-sional Jeffrey radiating nanofluid flow past stretching surface. J. Comput. Appl. Res. Mech. Eng. (JCARME) 2021. [CrossRef]

23. Shahzad, F.; Jamshed, W.; Nisar, K.S.; Khashan, M.M.; Abdel-Aty, A.-H. Computational analysis of Ohmic and viscous dissipation effects on MHD heat transfer flow of -PVA Jeffrey nanofluid through a stretchable surface. Case Stud. Therm. Eng. 2021, 26, 101148. [CrossRef] 
24. Rasool, G.; Shafiq, A.; Durur, H. Darcy-Forchheimer relation in Magnetohydrodynamic Jeffrey nanofluid flow over stretching surface. Discret. Contin. Dyn. Syst.-S 2021, 14, 2497. [CrossRef]

25. Rasheed, H.U.; Al-Zubaidi, A.; Islam, S.; Saleem, S.; Khan, Z.; Khan, W. Effects of Joule Heating and Viscous Dissipation on Magnetohydrodynamic Boundary Layer Flow of Jeffrey Nanofluid over a Vertically Stretching Cylinder. Coatings 2021, 11, 353. [CrossRef]

26. Ishak, A.; Nazar, R.; Pop, I. Boundary layer flow and heat transfer over an unsteady stretching vertical surface. Meccanica 2008, 44, 369-375. [CrossRef]

27. Vajravelu, K.; Prasad, K.V.; Ng, C.-O. Unsteady convective boundary layer flow of a viscous fluid at a vertical surface with variable fluid properties. Nonlinear Anal. Real World Appl. 2013, 14, 455-464. [CrossRef]

28. Kumar, R.N.; Jyothi, A.; Alhumade, H.; Gowda, R.P.; Alam, M.M.; Ahmad, I.; Gorji, M.; Prasannakumara, B. Impact of magnetic dipole on thermophoretic particle deposition in the flow of Maxwell fluid over a stretching sheet. J. Mol. Liq. 2021, $334,116494$. [CrossRef]

29. Gowda, R.P.; Al-Mubaddel, F.S.; Kumar, R.N.; Prasannakumara, B.; Issakhov, A.; Rahimi-Gorji, M.; Al-Turki, Y.A. Computational modelling of nanofluid flow over a curved stretching sheet using Koo-Kleinstreuer and Li (KKL) correlation and modified Fourier heat flux model. Chaos Solitons Fractals 2021, 145, 110774. [CrossRef]

30. Mallikarjuna, H.B.; Nirmala, T.; Gowda, R.J.P.; Manghat, R.; Kumar, R.S.V. Two-dimensional Darcy-Forchheimer flow of a dusty hybrid nanofluid over a stretching sheet with viscous dissipation. Heat Transf. 2021, 50, 3934-3947. [CrossRef]

31. McDade, J.; Phillips, G.; Sivinski, H.; Whitfield, W. Chapter V Principles and Applications of Laminar-flow Devices. Methods Microbiol. 1969, 1, 137-168. [CrossRef]

32. Everts, M.; Bhattacharyya, S.; Bashir, A.I.; Meyer, J.P. Heat transfer characteristics of assisting and opposing laminar flow through a vertical circular tube at low Reynolds numbers. Appl. Therm. Eng. 2020, 179, 115696. [CrossRef]

33. Makinde, O.D.; Sandeep, N.; Ajayi, T.M.; Animasaun, I.L. Numerical Exploration of Heat Transfer and Lorentz Force Effects on the Flow of MHD Casson Fluid over an Upper Horizontal Surface of a Thermally Stratified Melting Surface of a Paraboloid of Revolution. Int. J. Nonlinear Sci. Numer. Simul. 2018, 19, 93-106. [CrossRef]

34. Kumar, R.N.; Gowda, R.P.; Prasanna, G.; Prasannakumara, B.; Nisar, K.S.; Jamshed, W. Comprehensive study of thermophoretic diffusion deposition velocity effect on heat and mass transfer of ferromagnetic fluid flow along a stretching cylinder. Proc. Inst. Mech. Eng. Part E J. Process. Mech. Eng. 2021. [CrossRef]

35. Gowda, R.J.P.; Rauf, A.; Kumar, R.N.; Prasannakumara, B.C.; Shehzad, S.A. Slip flow of Casson-Maxwell nanofluid confined through stretchable disks. Indian J. Phys. 2021, 1-9. [CrossRef]

36. Makinde, O.D.; Olanrewaju, P.O. Unsteady mixed convection with soret and dufour effects past a porous plate moving through a binary mixture of chemically reacting fluid. Chem. Eng. Commun. 2011, 198, 920-938. [CrossRef]

37. Gowda, R.J.P.; Kumar, R.N.; Rauf, A.; Prasannakumara, B.C.; Shehzad, S.A. Magnetized flow of sutterby nanofluid through cattaneo-christov theory of heat diffusion and stefan blowing condition. Appl. Nanosci. 2021, 1-10. [CrossRef]

38. Woods, L.C. The Thermodynamics of Fluid Systems; Oxford University Press: New York, NY, USA; Clarendon Press: Oxford, UK, 1975; 371p, Available online: https:/ / ui.adsabs.harvard.edu/abs/1975cp...book.....W/abstract (accessed on 6 August 2021).

39. Yamaguchi, H. Engineering Fluid Mechanics; Springer: Dordrecht, The Netherlands, 2008. [CrossRef]

40. Malashetty, M.; Shivakumara, I.; Kulkarni, S. The onset of Lapwood-Brinkman convection using a thermal non-equilibrium model. Int. J. Heat Mass Transf. 2005, 48, 1155-1163. [CrossRef] 